\title{
SELECTION OF VISCOUS DAMPING COEFFICIENTS USING THE CONTINUOUS WAVELET TRANSFORM METHOD FOR A GEAR SYSTEM
}

\author{
Nourhaine Yousfi \\ Mechanics, Modelling and Production Research Laboratory (LA2MP), National Engineering School of Sfax, \\ University of Sfax, Tunisia \\ National Engineering School of Tunis, University of Tunis El-Manar, Tunisia \\ e-mail: nourhainegem@gmail.com
}

BACEM ZGHAL

Mechanics, Modelling and Production Research Laboratory (LA2MP), National Engineering School of Sfax, University of Sfax, Tunisia

Ali Akrout

Mechanics, Modelling and Production Research Laboratory (LA2MP), National Engineering School of Sfax, University of Sfax, Tunisia

National Engineering School of Tunis, University of Tunis El-Manar, Tunisia

LassaAd Walha, Mohamed Haddar

Mechanics, Modelling and Production Research Laboratory (LA2MP), National Engineering School of Sfax, University of Sfax, Tunisia

\begin{abstract}
Viscous damping is frequently used in the equation of motion to present the dissipation mechanism of a mechanical system. The orders of frequencies can be easily selected to determine viscous damping coefficients (VDCs) when the degree of freedom of the structure is low. For complex structures, difficulties in selecting the orders of reference frequencies to obtain reasonable VSCs are encountered. This paper mainly discusses the capability of the CWT method to select optimum frequencies of viscous damping formulation. The proposed procedure considers both the classical Rayleigh, modal and the proposed full model modal damping. The method is validated using a numerical time domain response of a two-stage gear system.
\end{abstract}

Keywords: viscous damping formulation, optimum frequencies, continuous wavelet transforms, dominant frequency

\section{Introduction}

Damping plays an important role in simulation of mechanical systems. Various models of damping are used such us non-viscous damping, Coulomb damping and hysteresis damping (Caughey and O'Kelly, 1965; Zhang and Wang, 2010). Non-viscous damping model is usually used in gear systems analysis. Recently, we presented a new technique to identify non-viscous damping in a one stage system using the integral method (Yousfi et al., 2018). However, for mathematical convenience, the viscous damping model is the most used formulation of damping because of its excellent advantages ( $\mathrm{Li}$ et al., 2017). Various forms of the viscous damping formulation have been implemented, which are simplified, full and extended Rayleigh formulations. These formulations require defining one or more coefficients, respectively (Park and Hashash, 2004). For gear system analysis, the damping ratio of the first mode is always used chosen to identify viscous damping parameters (Khabou et al., 2011; Yassine et al., 2014). However, more than 
one mode usually exists in a gear system with multi-DOF, and different modes give different results for viscous damping parameters (Chowdhury and Dasgupta, 2003b; Pan, 2013) so that this type of approach gives unsuitable results and has some limitations (Chopra, 2012; Tamura and Suganuma, 1996). Therefore, the frequencies of viscous damping formulation should be selected to overcome this problem. In other fields, such as in structural dynamics in earthquake engineering, many references to damping can be found. Chowdhury and Dasgupta (2003a) present a technique to identify viscous damping coefficients (VDCs) for systems with large degrees of freedom but their study neglects the influence of input motion to select the optimal frequencies of VDCs. Léger and Dussault (1992) used a Rayleigh damping model to study the dynamic response of a multi-degree-of-freedom structure, and affirmed that the damping formulations based on mass proportional, stiffness proportional and Rayleigh damping matrix represent different energy-consumption modes. Kwok et al. (2007) compared the calculated responses using a range of VDCs. It is demonstrated that the use of the 1-st and 5-th modes of the soil column is a good preliminary estimate. Pan et al. (2017) proposed a constrained optimization method to determine the exact damping ratio of modes that greatly contribute to dynamic responses. Li et al. (2015) developed an improved method to calculate Rayleigh damping coefficients by considering spectrum characteristics of the dynamic response and frequency characteristics of the structure. However, more fine tuning and additional trial-and-error estimates may be required to obtain the optimum frequencies of viscous damping formulation in the gear system. To calculate viscous damping coefficients, Chopra (2012) suggested that "in dealing with practical problems, it is reasonable to select the modes of vibrations $i$ and $j$ with specific damping ratios to ensure that the damping ratios of all modes of vibration that contribute greatly to the dynamic response are reasonable." Since, the means to identify damping ratios of a multi-DOF structure have advanced from the time-domain logarithm decay method and the frequency-domain half-power bandwidth method to the time-frequency domain wavelet transform method (Tamura and Suganuma, 1996; Reda Taha et al., 2006). The continuous wavelet transform method (CWT) is used in this study to select optimum modes of viscous damping formulation that greatly contribute to dynamic responses. This paper is organized as follows. Section 2 introduces the current viscous damping formulation. Section 3 provides the proposed calculation method of viscous damping coefficients. Section 4 presents validation of the proposed method using a numerical example of a two spur gear system. The modes that remarkably affect the dynamic responses are determined on the basis of the mode corresponding to the maximum value of the wavelet spectrum, and the Rayleigh damping coefficients are calculated and the results of the dynamic response obtained by different methods are presented and compared. Numerical results show that the proposed method accurately reveals the modes contributing to the dynamic response of the two-stage gear system, and the calculated VDCs are consistent with actual results. The calculated results are also closer to the exact solutions and even higher than those acquired by traditionnal methods. Section 5 provides the conclusion.

\section{Current viscous damping formulation}

In mechanical systems, the following dynamic equation of motion is solved

$$
\mathbf{M} \ddot{\mathbf{q}}+\mathbf{C} \dot{\mathbf{q}}+\mathbf{K q}=\mathbf{f}_{0}
$$

where: $\mathbf{M}$ - mass matrix, $\mathbf{C}$ - viscous damping matrix, $\mathbf{K}$ - stiffness matrix. The dynamic equilibrium equation, Eq. (2.1), is solved at each time using a step by step iterative Newmark method. The damping matrix $\mathbf{C}$ which is better represented by hysteretic models where the dissipation force is proportional to displacement or friction. However, viscous damping is widely 
considered as an equivalent approach because it leads to simple mathematical treatment. Various forms of the viscous damping formulation have been implemented, but the most common viscous damping formulation description is the uniform Rayleigh model which is derived from a combination of the mass matrix and the stiffness matrix (Rayleigh et al., 1945)

$$
\mathbf{C}=\alpha \mathbf{M}+\beta \mathbf{K}
$$

The coefficients $\alpha$ and $\beta$ in Eq. (2.2) are defined as follows

$$
\alpha=\xi \frac{4 \pi\left(f_{0} f_{1}\right)}{f_{0}+f_{1}} \quad \beta=\xi \frac{1}{\pi\left(f_{0}+f_{1}\right)}
$$

where $\xi$ is the target damping ratio. Equation (2.3) matches the target damping ratio only at two frequencies, $f_{0}$ and $f_{1}$. However, it is unrealistic to assume a constant damping ratio for all modes, and the damping formulation will filter out a significant portion of the high frequency content of the dynamic response. So a more fine procedure may be required to obtain the optimum frequencies of the Rayleigh damping formulation. In the modal basis, modal damping corresponds to the assumption that the viscous damping matrix is diagonal, that is to say that

$$
\boldsymbol{\Phi}^{\mathrm{T}} \mathbf{C} \boldsymbol{\Phi}=\left[\begin{array}{lll}
\ddots & & \\
& 2 w_{j} \xi_{j} & \\
& & \ddots
\end{array}\right]
$$

where $\boldsymbol{\Phi}$ is the modal matrix normalized, the real modeshapes $\phi_{j}$ and pulsations $w_{j}$ are solutions of the eigenvalue problem

$$
\left(-w_{j}^{2} \mathbf{M}+\mathbf{K}\right) \phi_{j}=\mathbf{0}
$$

Using the mass orthogonality conditions and the fact that a matrix and its inverse can be permuted, the following relations are obtained

$$
\mathbf{C}=[\mathbf{M} \boldsymbol{\Phi}]_{N \times N}\left[\begin{array}{lll}
\ddots & & \\
& 2 w_{j} \xi_{j} & \\
& & \ddots
\end{array}\right]_{N \times N}[\mathbf{M} \boldsymbol{\Phi}]_{N \times N}^{\mathrm{T}}
$$

In the current modal damping, Equation (2.8) is exact when all modes are taken into account.

\section{Proposed calculation method of viscous damping coefficients}

\subsection{Proposed Rayleigh damping and modal damping}

In the original formulation proposed by Rayleigh and Lindsay (Rayleigh et al., 1945), $\alpha$ and $\beta$ coefficients in Eq. (2.2) can be determined using two natural modes $m$ and $n$

$$
\alpha=\frac{2 w_{k} w_{n}}{w_{n}^{2}-w_{k}^{2}}\left(\xi_{k} w_{n}-\xi_{n} w_{k}\right) \quad \beta=\frac{2\left(\xi_{n} w_{n}-\xi_{k} w_{k}\right)}{w_{n}^{2}-w_{k}^{2}}
$$

where $w_{n}$ and $w_{k}$ corresponds to the circular frequency of the $n$-th and $k$-th modes and their damping ratios $\xi_{n}$ and $\xi_{k}$ obtained through measurement. The constants $\alpha$ and $\beta$ are obtained by specifying the desired damping ratios of two different modes and solving a system of two equations and two unknowns at two different preselected frequencies. Therfore, this study presents an initial use of the formulation of Rayleigh damping presented in Eq. (3.1) in the gear system 
to model the damping matrix by specifying the desired damping ratios of two different modes using the wavelet transform. In the proposed modal damping presented in this paper, only a modal series of $N_{m}$ modes is used. To carry out this simplification it is important to realize that the rows of $\boldsymbol{\Phi}^{-1}$ are given by $\boldsymbol{\phi}_{j}^{\mathrm{T}} \mathbf{M}$ to obtain a closed form solution of the modal damping matrix (Bianchi et al., 2010)

$$
\mathbf{C}=[\mathbf{M} \Phi]_{N \times N m}\left[\begin{array}{lll}
\ddots & & \\
& 2 w_{j} \xi_{j} & \\
& & \ddots
\end{array}\right]_{N m \times N m}[\mathbf{M} \boldsymbol{\Phi}]_{N m \times N}^{\mathrm{T}}
$$

In traditional studies, the number $N_{m}$ of modes is selected using time domain analysis. However, the number $N_{m}$ of modes and the associated damping coefficients directly impact computation time and generally require a presentation of time-frequency maps. This paper presented initial investigation into the use of the Continious Wavelet Transform (CWT) method to provide superior time-frequency analysis and to characterize the optimal modes $N_{m}$ out in a modal truncation procedure. The following Section presents the CWT technique and different steps established to select VDCs.

\subsection{Proposed selection method}

The CWT-based damping-identification procedure is composed by the following steps. Initially, the signal of the gear system should be transformed at the time-frequency plot using Eq. (3.3). The WT of a signal $y(t)$ is defined by

$$
\operatorname{CWT}\{y(t)\}=W_{x}(\tau, b)=\frac{1}{\sqrt{b}} \int_{-\infty}^{+\infty} y(t) \Psi^{*}\left(\frac{t-\tau}{b}\right) d t
$$

where $\Psi^{*}(t)$ is the complex conjugate of $\Psi(t)$. A wavelet plot can be obtained which presents the modulus of the wavelet coefficients $\left|W_{x}(\tau, b)\right|$ in the time $\tau$ and the scale $b$, which is important to correctly obtain relevant time-frequency resolutions. The second step is the ridge detection which represents the high energy density. The third step is to identify the damping ratio using the logarithmic decay of the envelope of each mode separately using the following equation

$$
\ln \left|w_{x}\left(a_{0}, b\right)\right|=\xi \omega_{n} b+\ln \left(\frac{\sqrt{a_{0}}}{2} B\left|\Psi^{*}\left(a_{0}, \omega_{d}\right)\right|\right)
$$

The last step of the CWT-based damping-identification is to determine the dominant frequencies from which the optimum frequencies of the viscous damping formulation are selected. For the proposed Rayleigh damping model, the proposed procedure uses the 1-st mode for $w_{k}$ and defines $w_{n}$ based on the range of frequency at which the high amplitude of wavelet transform is concentrated, which is determined from the wavelet plot. For the proposed modal damping, the selection of the trunced frequencies $N_{m}$ is based on the dominant frequencies observed on the wavelet plot. The FFT method is used to determine the rest of the frequencies then, the CWT method is used to determine the damping ratio of each frequency. The steps of the proposed method are presented in Fig. 1.

In Section 4, the CWT method is used to analyze the uncertainties resulted from usage of classical Rayleigh and modal damping which can not predict the real response of the system and to examine the proposed viscous damping with selected frequencies based on the CWT to overcome this problem. The performance of the proposed method is validated by a simulated example of the two-stage gear system. 


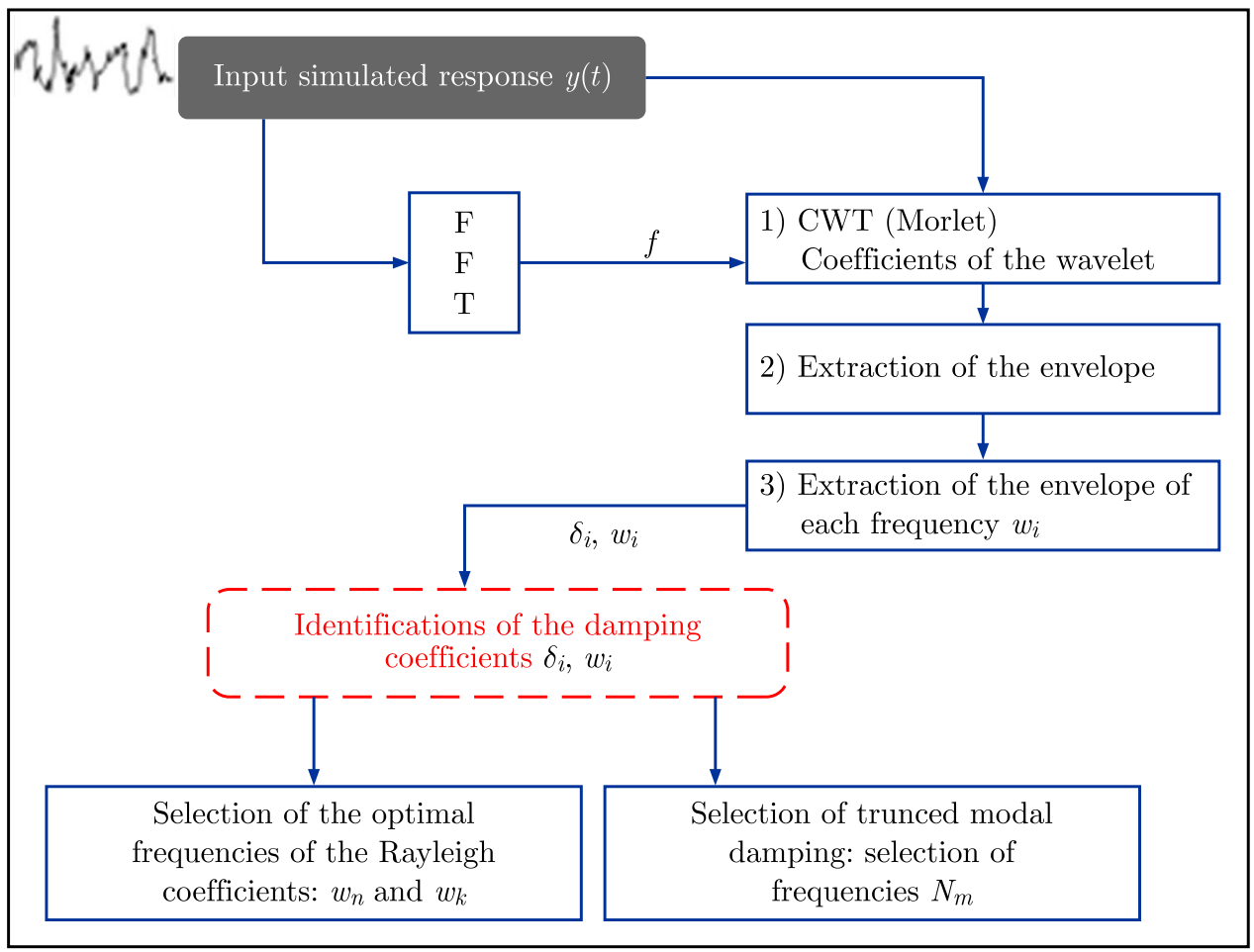

Fig. 1. Steps of the wavelet transform method

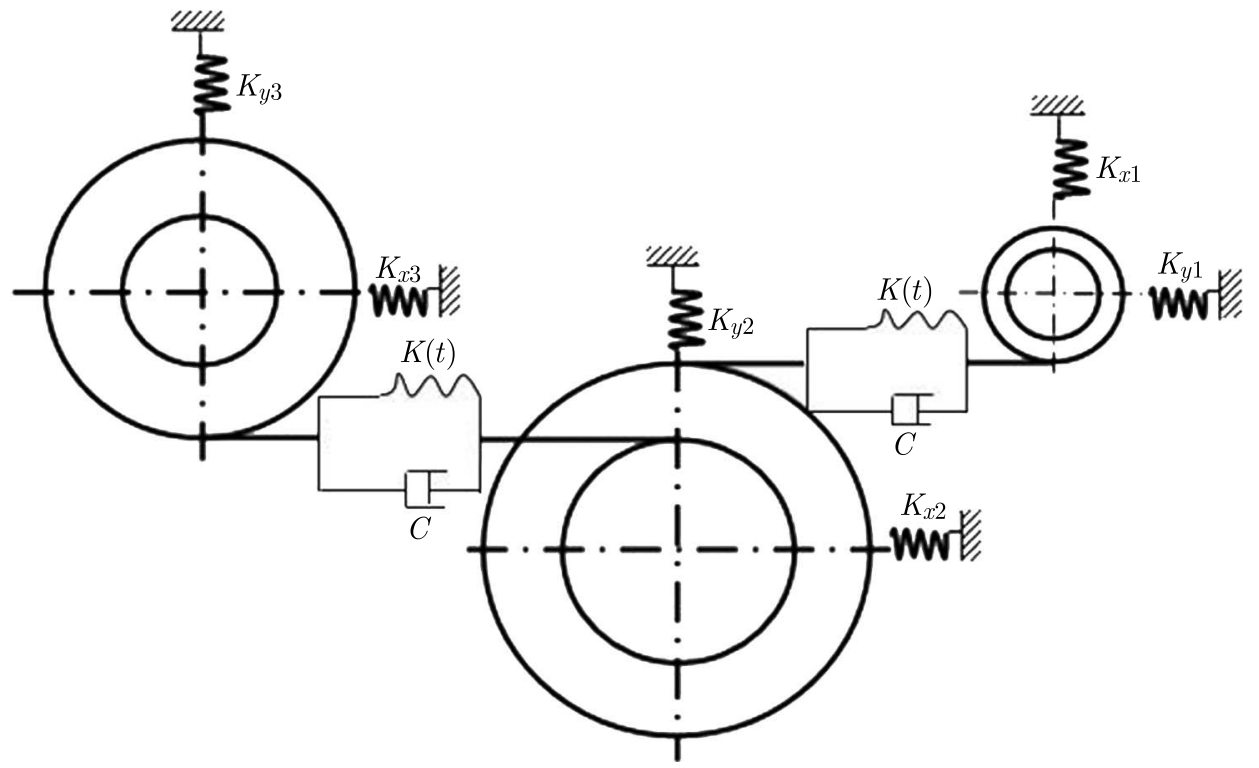

Fig. 2. Dynamic model of the two-stage spur gear system

\section{Numerical example and validation of the proposed algorithm}

\subsection{Numerical example}

The dynamic behavior of the gear system can be examinated in terms of the transmission error. In Fig. 2, the dynamic model of a two-stage gear system is established with the parameters presented in Table 1 (Walha et al., 2009). The gears are modelled by concentrated masses, bearings are modelled by linear springs in the gears plans. The shafts are supposed flexible with neglected masses. The dynamic model studied in this paper is characterised by 12 degrees of freedom and can be defined by 


$$
\mathbf{q}=\left[x_{1}, y_{1}, x_{2}, y_{2}, x_{3}, y_{3}, \theta_{m}, \theta_{1}, \theta_{2}, \theta_{3}, \theta_{r}\right]
$$

where $x_{j}$ and $y_{j}$ are bearings displacements, $\theta_{i}(i=1, \ldots, 4)$ are dynamic angular displacements of the gears, $\theta_{m}$ and $\theta_{r}$ are dynamic angular displacements of the motor and brake.

The two teeth deflections $\delta_{1}(t)$ and $\delta_{2}(t)$ of each stage gear are defined by

$$
\begin{aligned}
& \delta_{1}(t)=\left(x_{1}-x_{2}\right) \sin \alpha_{1}+\left(y_{1}-y_{2}\right) \cos \alpha_{1}+r_{1} \theta_{1}-r_{2} \theta_{2} \\
& \delta_{2}(t)=\left(x_{2}-x_{3}\right) \sin \alpha_{2}+\left(y_{2}-y_{3}\right) \cos \alpha_{2}+r_{3} \theta_{3}-r_{4} \theta_{4}
\end{aligned}
$$

Lagrange formulation was used to formulate the differential equation governing motion of the two-stage gear system. The general matrix form of this 12 degrees of freedom system is defined by

$$
\mathbf{M} \ddot{\mathbf{q}}+\mathbf{C} \dot{\mathbf{q}}+\left[\mathbf{K}_{s}+\mathbf{K}(t)\right] \mathbf{q}=\mathbf{F}_{0}
$$

where the external excitation vector of the system is

$$
\mathbf{F}_{0}=[0,0,0,0,0,0, C m, 0,0,0,0,-C r]^{\mathrm{T}}
$$

and $\mathbf{M}$ is the mass matrix. In most previous works (Walha et al., 2009), viscous damping has been considered to reduce the level of vibrations given by

$$
\mathbf{C}=\alpha \mathbf{M}+\beta \mathbf{K}_{m o y}
$$

where $\mathbf{K}_{m o y}$ is the average stiffness matrix of the system (Walha et al., 2009). $\alpha$ and $\beta$, respectively, represent the mass and stiffness proportional damping coefficients which are collectively known as VScs. The resolution of each linear system is made thanks to a step by step iterative Newmark method (Dhatt and Touzot, 1984). The resolution of Eq. (3.7) gives the simulated responses presented in Fig. 3 which corespond to the first teeth deflection fluctuation and the second teeth deflection fluctuation following the time. The performance of the proposed VSCs identification method using CWT presented in this paper is validated based on the simulated examples presented in Fig. 3. Our objective is to identify the VSCs from only the simulated responses (the input first teeth deflection and the input second teeth deflection) of Fig. 3 and its wavelet transform.

Table 1. Parameters of the studied two-stage gear system (Walha et al., 2009)

\begin{tabular}{|l|c|}
\hline Material: $42 \mathrm{CrMo} 4$ & $\rho=7860 \mathrm{~kg} / \mathrm{m}^{3}$ \\
\hline Motor torque $[\mathrm{Nm}]$ & $C m=10$ \\
\hline Bearings stiffnesses $[\mathrm{N} / \mathrm{m}]$ & $k x_{i}=k y_{i}=10^{7}$ \\
\hline Torsional shaft flexibilities $[\mathrm{Nm} / \mathrm{rad}]$ & $k \theta_{i}=10^{5}$ \\
\hline Pressure angle & $\alpha_{1}=\alpha_{2}=20^{\circ}$ \\
\hline Teeth module $[\mathrm{m}]$ & $m=4 \cdot 10^{-3}$ \\
\hline Teeth number & $Z_{1}=30, Z_{2}=45, Z_{3}=30, Z_{4}=45$ \\
\hline Average mesh stiffness $[\mathrm{N} / \mathrm{m}]$ & $k_{1 \text { moy }}=k_{2 \text { moy }}=3 \cdot 10^{7}$ \\
\hline Contact ratio & $\varepsilon_{\alpha 1}=\varepsilon_{\alpha 2}=1.592$ \\
\hline
\end{tabular}

\subsection{Validation of the proposed method}

In order to validate the usefulness of the wavelet transform method in modal analysis to select optimum frequencies/modes of the viscous damping formulation, a suite of analysis is performed on the simulated responses of the two-stage gear system presented in Fig. 2. There 
(a)

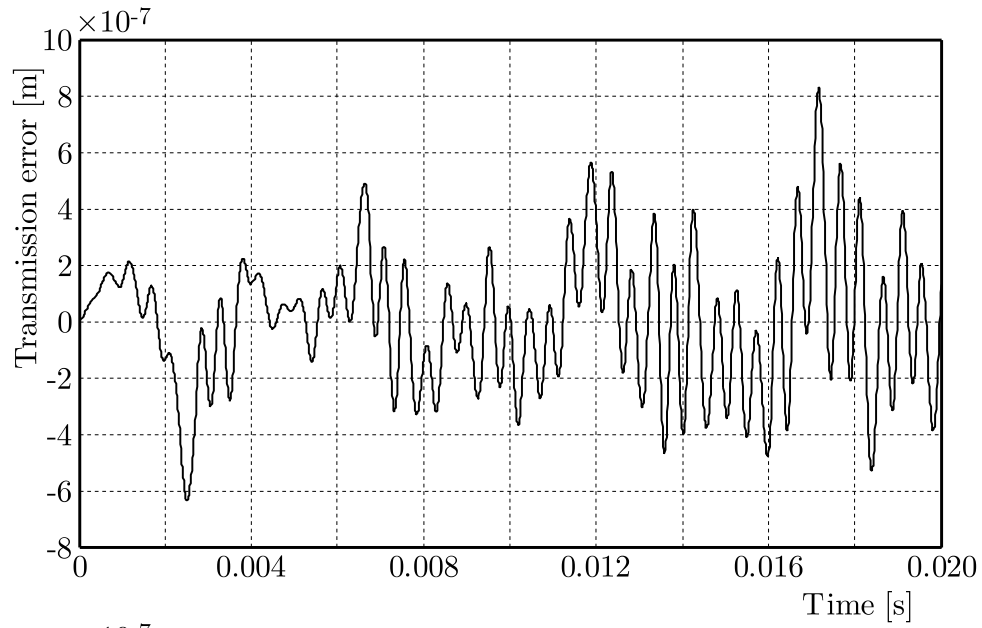

(b)

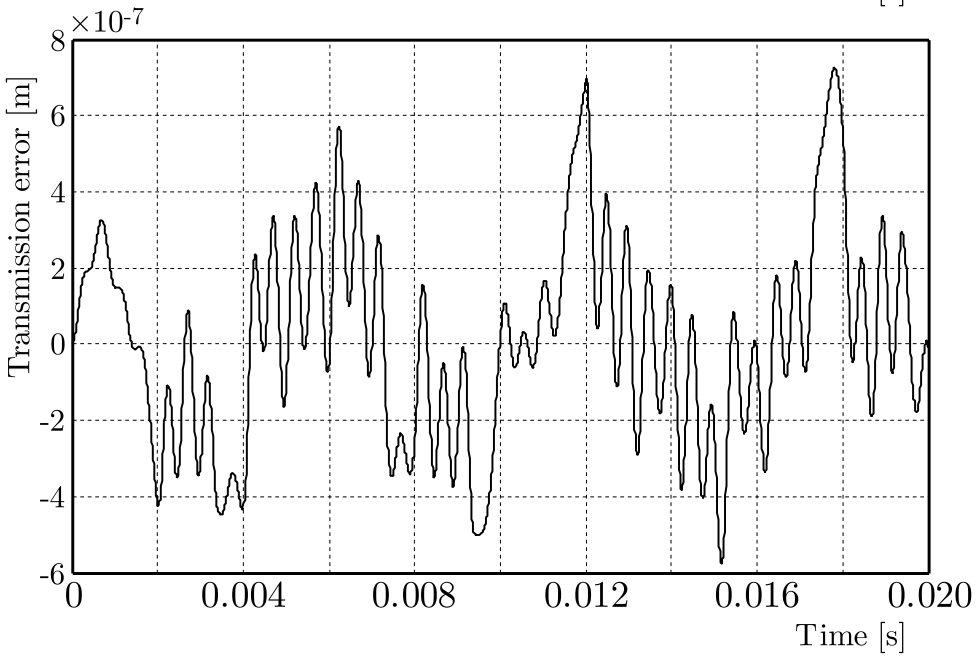

Fig. 3. (a) Temporal first teeth deflection, (b) temporal second teeth deflection

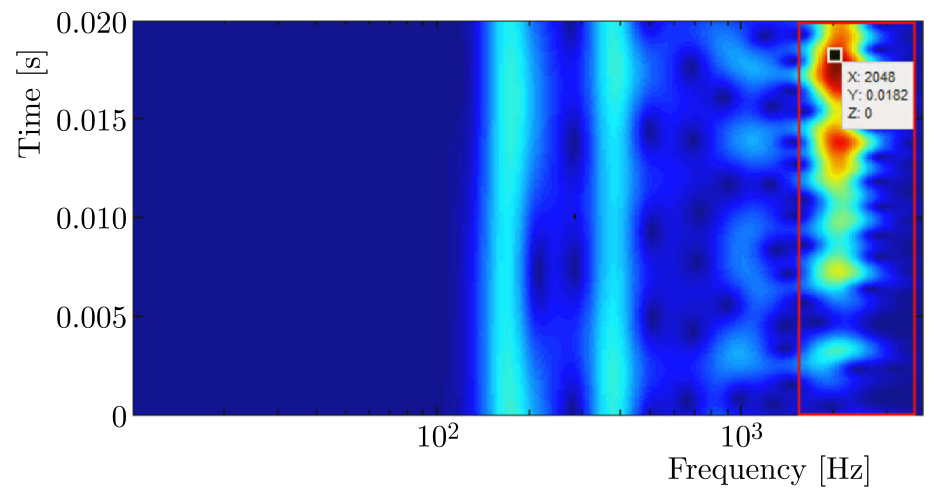

Fig. 4. Wavelet plot of the first teeth deflection signal

are two models considered for the system which are Rayleigh damping and modal damping. The input dynamic response $s$ of the first stage and the second stage used in the analyses are shown in Figs. 3a and 3b. To estimate the modal parameters, we use the steps described in Section 3. Figure 4 shows the energy spectrum distribution in the time-frequency domain of the first stage; we obtain a superior time-frequency analysis of the simulated response and the presented modes can be easily observed from this plot, in particular from Fig. 5a. Seven values of the dilatation parameter are predominant in this plot. They correspond to the number of modes present in the frequency band. The 7-th mode corresponds to the dominant frequency 
(a)

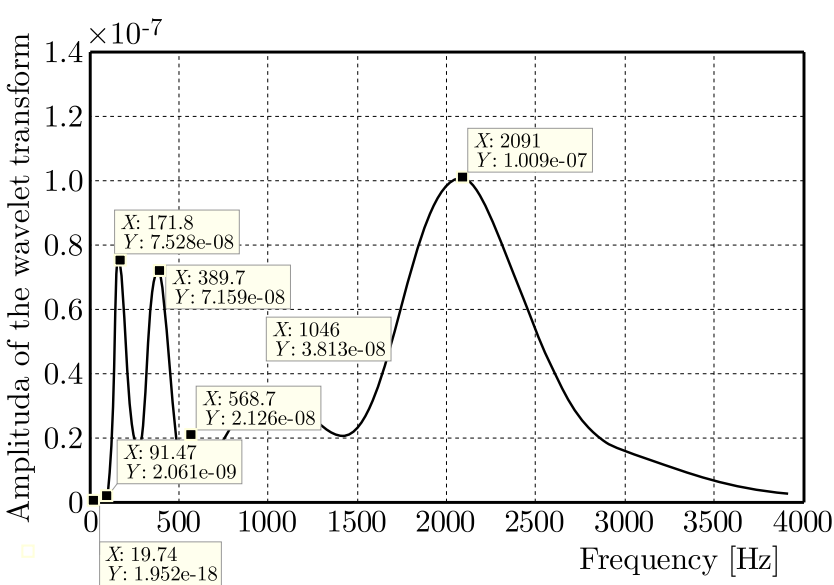

(b)

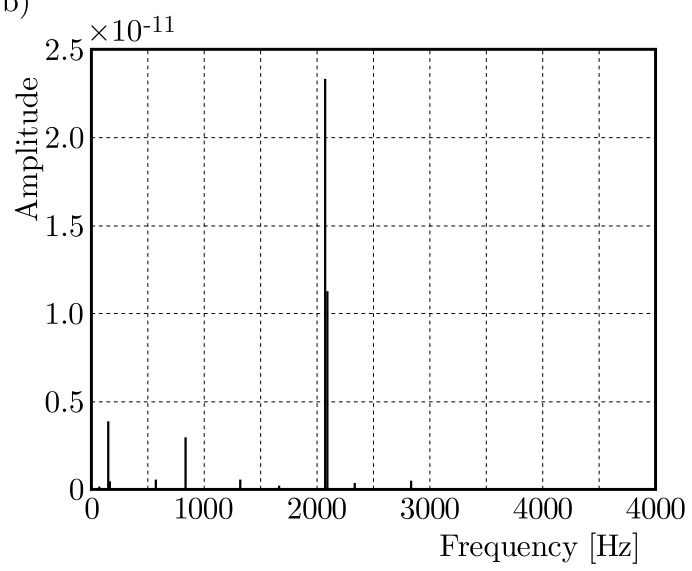

Fig. 5. (a) Power spectrum of the first teeth deflection signal using CWT method by a slice parallel to the time axis $[0,0.02]$, (b) power spectrum of the first teeth deflection signal using FFT method

where high energy is concentrated and used to determine the VDCs. The power spectrum of the second teeth deflection signal is found using the FFT method. The wavelet envelope can be extracted from the wavelet plot as it is shown in Fig. 6 by a slice parallel to the time axis through each frequency $w_{i}$ for $i=1, \ldots, 7$ in the frequency axis, which can be used to estimate the corresponding damping ratio. Thus the damping ratio of each mode can be estimated from the slope of the straight line of the wavelet modulus. The identified modal parameters for the first seven modes presented in Table 2 are obtained using Eq. (3.4) and from the plots shown in Fig. 6.

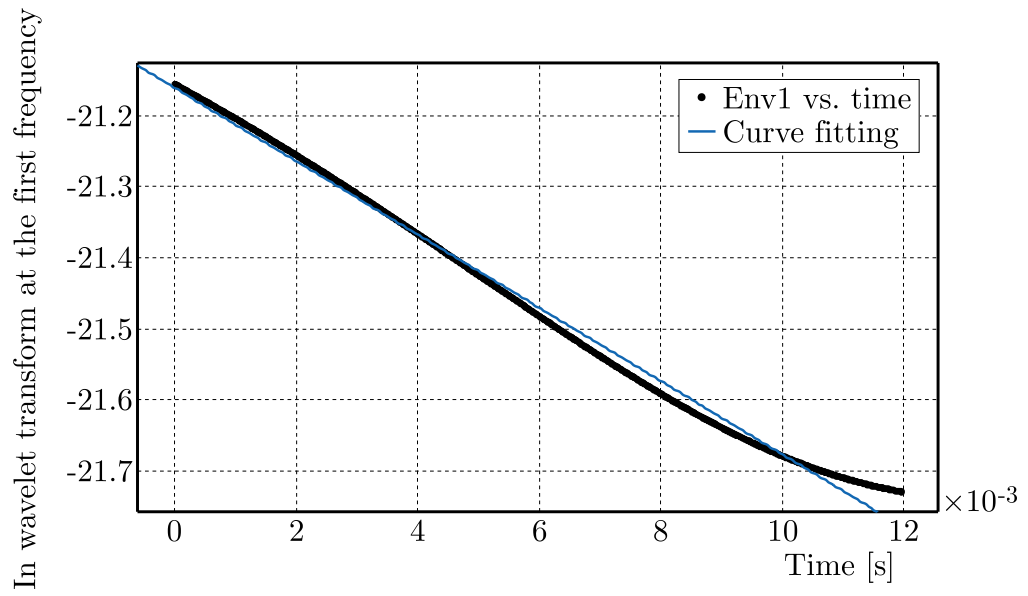

Fig. 6. The envelope of the wavelet ridge at the first natural frequency, -- signal, - - - linear regress

Table 2. Damping ratios corresponding to frequencies using Wavelet demodulation

\begin{tabular}{|c|c|c|c|c|c|c|c|}
\hline Parameter & $i=1$ & $i=2$ & $i=3$ & $i=4$ & $i=5$ & $i=6$ & $i=7$ \\
\hline \hline$f_{i}=w_{i} /(2 \pi)[\mathrm{Hz}]$ & 61.36 & 84.09 & 171.8 & 381.6 & 580.8 & 1046 & 2048 \\
\hline$\xi_{i}$ & 0.302854 & 0.036399 & 0.01546078 & 0.01022876 & 0.0178635 & 0.002389 & 0.00827 \\
\hline
\end{tabular}

The same steps of the wavelet transform procedure are applied to the second input teeth deflection presented in Fig. 3b. Figure 7 shows the energy spectrum distribution in the time-frequency domain of the second stage. We obtain an appropriate distribution of energy and the modes can be easily observed from this plot, in particular from Fig. 7. Six values of the 
dilatation parameter are predominant in this plot and they correspond to the number of modes present in the frequency band. The first mode and the 6-th mode correspond to the dominant frequency where high energy is concentrated. The wavelet envelope can be extracted from the wavelet plot as it is shown in Fig. 8 by a slice parallel to the time axis through each frequency $w_{i}$ for $i=1, \ldots, 6$ in the frequency axis, which can be used to estimate the corresponding damping ratio. The identified modal parameters for the first six modes presented in Table 3 are obtained using Eq. (3.4) and from the plots shown in Fig. 9. Thus the damping ratio of each mode can be estimated from the slope of the straight line of the wavelet modulus. Then, the predominant frequencies obtained using the wavelet transform are used to select the optimum frequencies of the viscous damping for each stage.

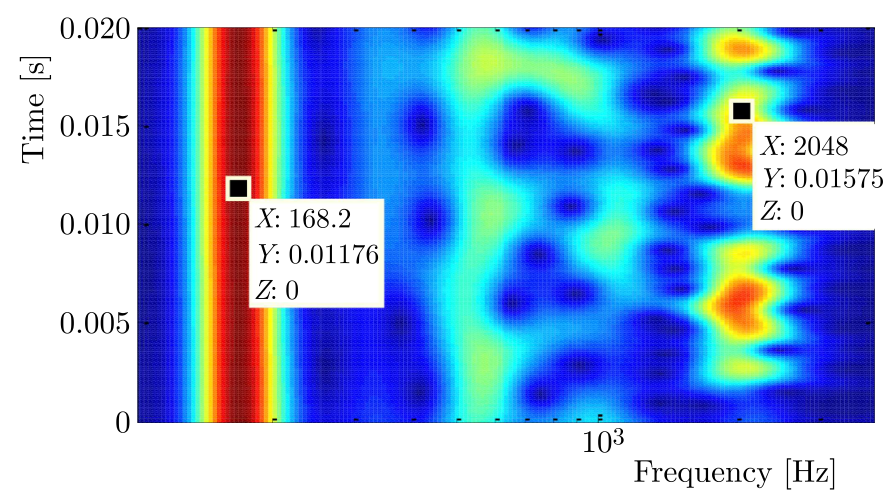

Fig. 7. Wavelet plot of the second teeth deflection signal

(a)

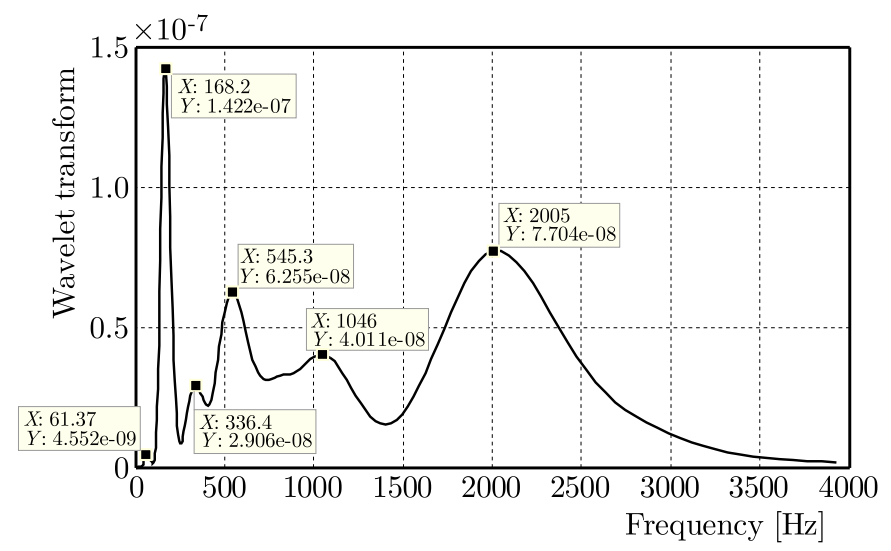

(b) $1.6 \times 10^{-11}$

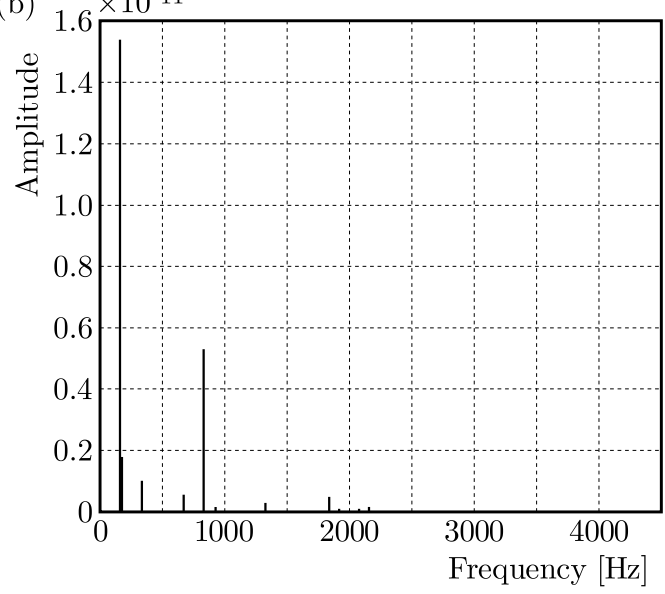

Fig. 8. (a) Power spectrum of the first teeth deflection signal using CWT method by a slice parallel to the time axis, (b) power spectrum of the first teeth deflection signal using FFT method

Table 3. Damping ratios corresponding to frequencies using wavelet demodulation

\begin{tabular}{|c|c|c|c|c|c|c|}
\hline Parameter & $i=1$ & $i=2$ & $i=3$ & $i=4$ & $i=5$ & $i=6$ \\
\hline \hline$f_{i}=w_{i} /(2 \pi)[\mathrm{Hz}]$ & 62.66 & 168.189 & 336.37 & 545.3 & 1045 & 2048 \\
\hline$\xi_{i}$ & 0.0327 & 0.0014 & 0.0394 & 0.0435 & 0.0271 & 0.0024 \\
\hline
\end{tabular}

Then, two models of viscous damping are investigated, which are Rayleigh damping and modal damping in the following Section for each stage. 


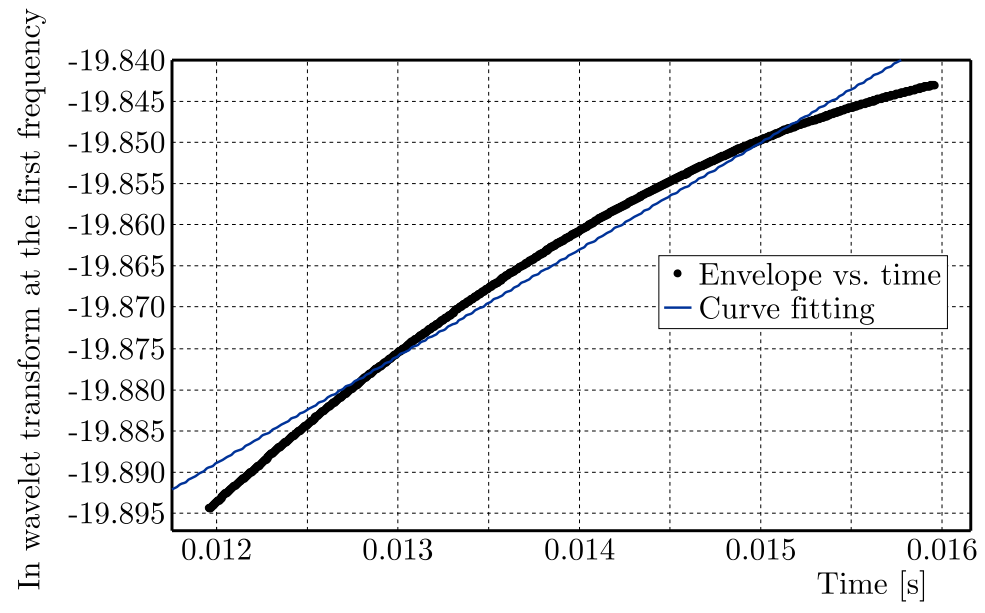

Fig. 9. The envelope of the wavelet ridge at the first natural frequency, -- signal, - - - linear regression

\subsubsection{Rayleigh damping}

Some codes are available in selecting the parameters of Rayleigh damping presented in Eq. (2.4), which is usually modeled by a target damping ratio $\xi(1 \%-5 \%)$ and not an optimum estimate for the frequencies $w_{k}$ and $w_{n}$. Thus, it results in ambiguities in the current practice because damping parameters should be selected through an iterative process depending on the characteristics of the input motion. Therefore, there is a need to develop a procedure which is capable of automatically selecting the coefficients for a given response of the system. This paper develops a new algorithm for selecting the coefficients of the Rayleigh damping formulation based on the calculated modal parameters obtained using the wavelet transform of the input response. The selected coefficients using CWT method are closely compared to the results obtained using the conventional Rayleigh formulations. Figures 10 and 12 compare the input teeth deflection signals of the two-stages of the gear system with the obtained response using different optimal frequencies and the conventional Rayleigh damping formulations.

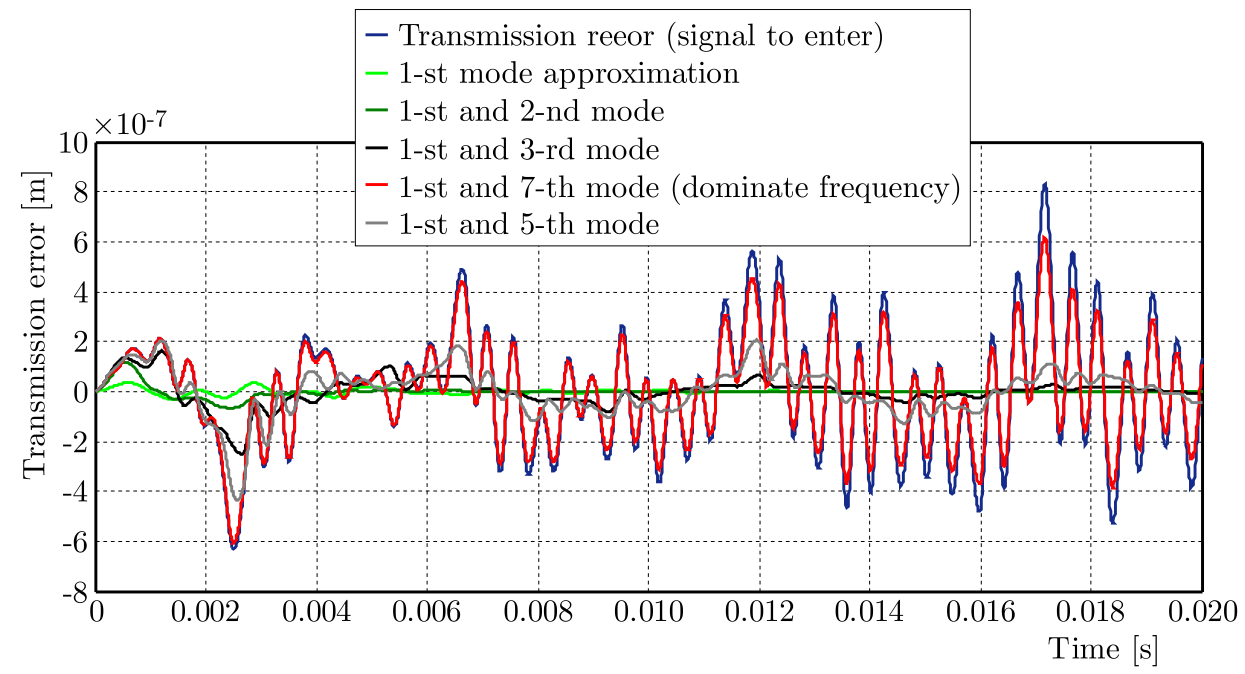

Fig. 10. First teeth deflection signal determined from the first natural mode approximation as well as proposed full Rayleigh viscous damping formulation

As it shown in Fig. 11, the conventional Rayleigh damping formulation filters out a significant portion of the response and filters out an important component at the high frequency range, and a good match is obtained when using $w_{k}$ as the first mode and $w_{n}$ as the dominant frequency 


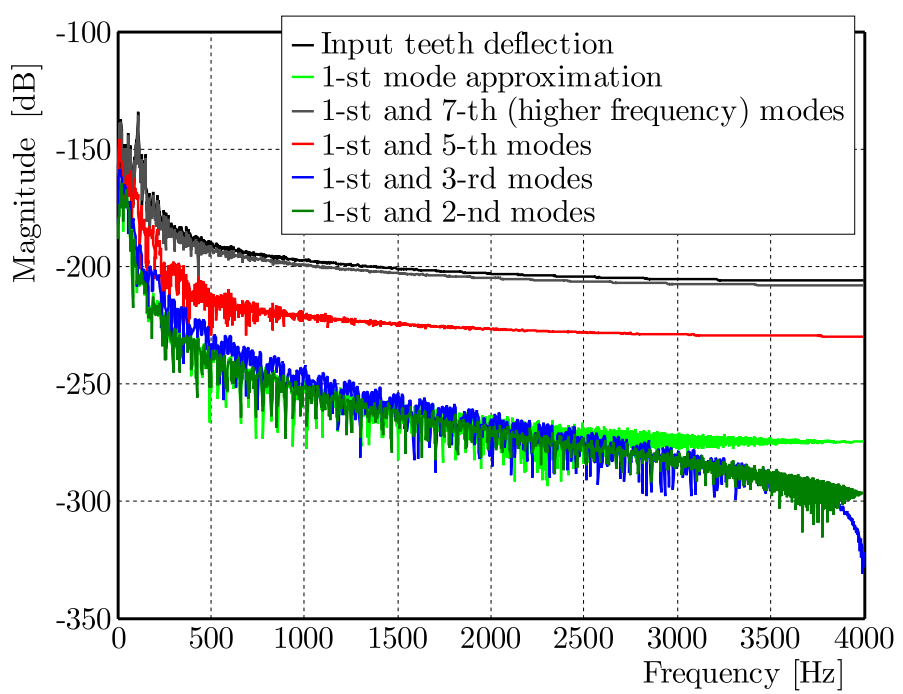

Fig. 11. Computed surface Fourier spectra of the first teeth deflection signal from first natural mode approximation as well as proposed full Rayleigh viscous damping formulation

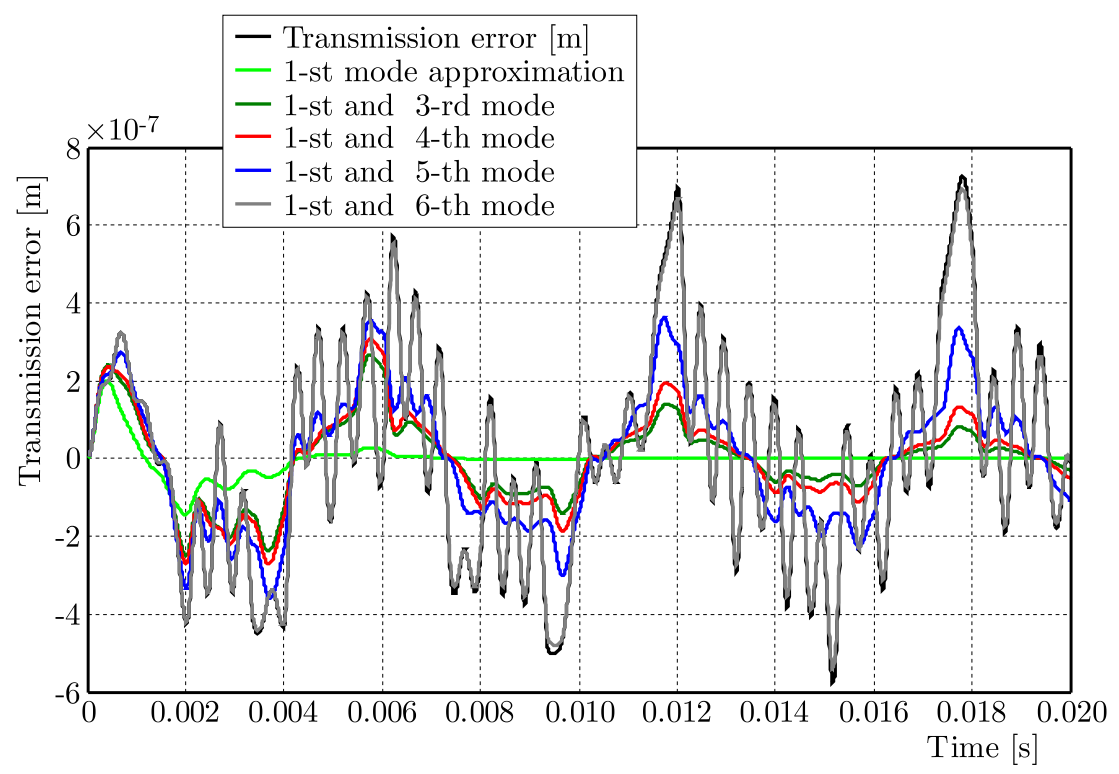

Fig. 12. Second teeth deflection signal found from the first natural mode approximation as well as proposed full Rayleigh viscous damping formulation

(higher mode), which corresponds the maximum value of the wavelet transform. Thus, the use of the proposed Rayleigh damping with the selected frequencies provides an exact match of the results from the time domain and frequency domain analysis. Therefore, the proposed Rayleigh damping formulation suggests that the first mode and the dominant mode (7-th mode for the first stage and 6-th mode for the second stage) obtained using the CWT method have an important contribution to the Rayleigh damping formulation. In the modal basis, the traditional practice is to use modal damping where the viscous damping matrix is diagonal of the form $2 \xi_{j} w_{j}$, where the number of modes must be selected to give the best match for the input deflection. In the following paragraph, the selection of modes of the modal damping is obtained using the CWT procedure. 


\subsubsection{Modal damping}

The numerical cost of modal damping is directly associated with the number of retained modes. Therfore, the CWT method provides a simple procedure to get modes that have influence on response analysis with respect to the minimum number of frequencies required for achieving the model of damping to fit global behavior better. A part of this study is thus to select target modes of the equation to obtain a proposed selective modal daming to compare it with the conventional modal damping where all modes are kept. The optimal frequencies $N_{m}$ should be selected as for the presented modes observed in the wavelet plot, 7 out of 12 modes are retained for the first stage and 6 modes are retained for the second stage. Figures 13 and 14 compare the input teeth deflection signal of the two-stages of the gear system with the obtained

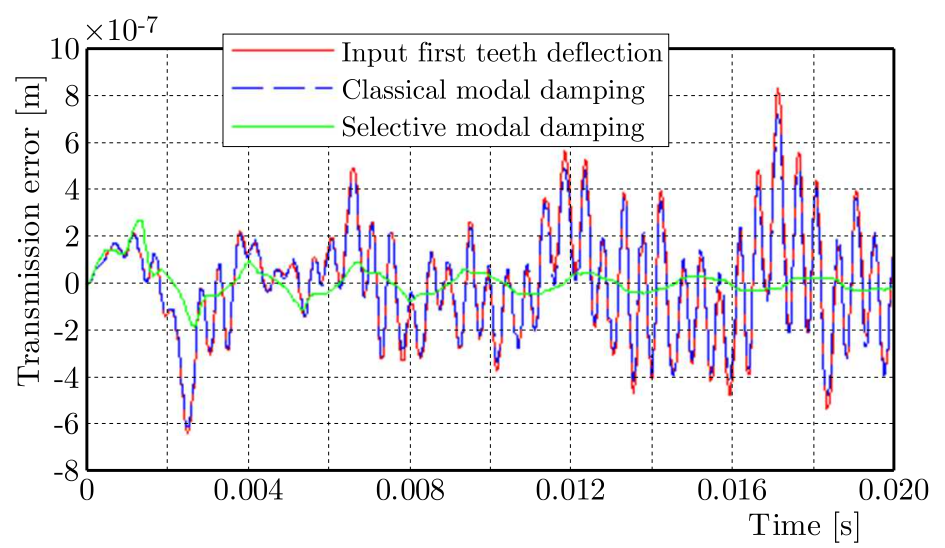

Fig. 13. First teeth deflection signal for classical modal damping (using all modes) as well as full modal damping formulation (using selective modes)

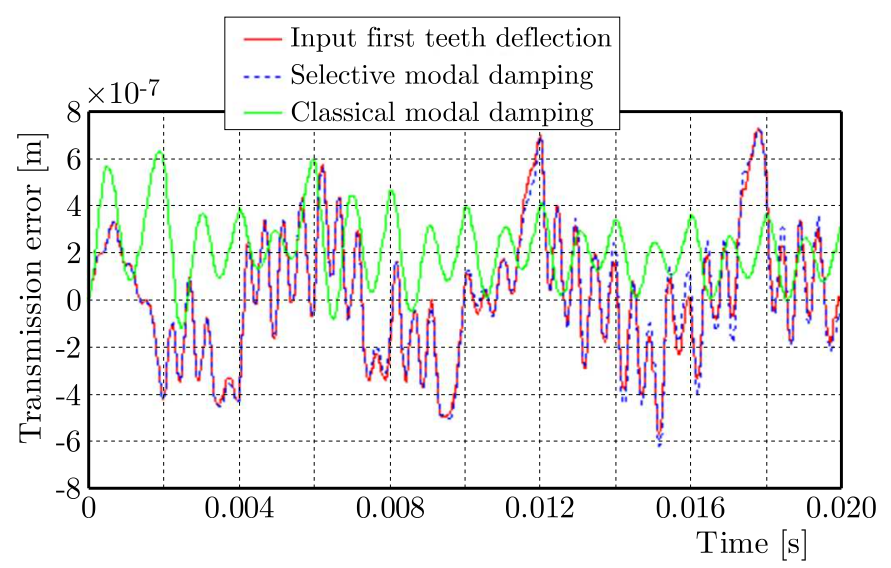

Fig. 14. Second teeth deflection signal for the first natural mode approximation as well as the proposed full Rayleigh viscous damping formulation

response using selective modal damping (the number of modes $N_{m}$ is selected using CWT) and the conventional modal damping formulation (all modes are kept). The results nearly match the input deflection for the two-stages and capture the peaks in low periods. At longer periods, the results obtained using the conventional damping underestimate the input deflection at periods more than $0.0025 \mathrm{~s}$, and a good match is obtained using the selective modal damping. From the results, we can conclude that the option of using the number of modes $N_{m}$ obtained using the CWT method (7-th modes for the first stage and 6-th modes for the second stage) lower than the total number of modes $N$ should be applied in lieu of the simplified modal damping (all modes are kept) to gave response characteristics that are close to the ones of the actual system. 
It must be noted that the rest of modes are obtained using the FFT method, and the resulting damping ratio for each mode is estimated using the CWT method.

\section{Conclusion}

The calculation methods of VDCs greatly affect dynamic responses of a two-stage gear system. As a result, the dynamic response is significantly smaller. In the method proposed in this study, the modes orders that influence the dynamic responses are found on the basis of the contunious wavelet trasform method, and VDCs are determined by using the natural frequencies of the mode orders where high energy is concentrated to minimize the effect of the frequency-dependent damping. In the proposed method, the teeth deflections are presented from a numerical example. Next, the test signals are treated by a complex Morlet CWT to identify the damping ratio related to each dominant frequency. Finally, analysis is performed to determine the optimal model of Rayleigh and modal damping.

Comparisons with the time domain response demonstrate that the proposed procedure is very effective in selecting the optimum frequencies from the input motion. Moreover, the estimated damping ratio and the Rayleigh and modal damping coefficients of the two-stage gear system may provide a suitable starting point for many codes to choose the optimum damping model.

\section{References}

1. Bianchi J.-P., Balmés E., Des Roches G.V., Bobillot A., 2010, Using modal damping for full model transient analysis: application to pantograph/catenary vibration, [In:] Proceedings of ISMA2010 - International Conference on Noise and Vibration Engineering Including, P. Sas, B. Bergen (Edit.), Leuven, Belgium, 20-22

2. Caughey T.K., O'Kelly M.E., 1965, Classical normal modes in damped linear dynamic systems, ASME Journal of Applied Mechanics, 32, 583-588

3. Chopra A.K., 2012, Dynamics of Structures: Theory and Applications to Earthquake Engineering, Prentice-Hall

4. Chowdhury I., Dasgupta S.P., 2003a, Computation of Rayleigh damping coefficients for large systems, Electronic Journal of Geotechnical Engineering, 43, 6855-6868

5. Chowdhury I., Dasgupta S.P., 2003b, Computation of Rayleigh damping coefficients for structures with large degrees of freedom, Electronic Journal of Geotechnical Engineering, 8, 1-11

6. Dhatт G., Touzot G., 1984, Finite Elements Method Presentation, Maloine Edition

7. Khabou M.T., Bouchaala N., Chaari F., Fakhfakh T., Haddar M., 2011, Study of a spur gear dynamic behavior in transient regime, Mechanical Systems and Signal Processing, 25, 8, 3089-3101

8. Kwok A.O.L., Stewart P.J., Hashash Y.M.A., Matasovic N., Pyke R., Wang Z., Yang Z., 2007, Use of exact solutions of wave propagation problems to guide implementation of nonlinear seismic ground response analysis procedures, Journal of Geotechnical and Geoenvironmental Engineering, 133, 1385-1398

9. LÉGer P., Dussault S., 1992, Seismic-energy dissipation in MDOF structures, Journal of Structural Engineering, 118, 1251-1270

10. Li Y., Sun Y., Li B., Xu Z., 2017, Penalty function-based method for obtaining a reliability indicator of gravity dam stability, Computers and Geotechnics, 19-25

11. Li Z., Wang G., Hu Y., Hu J.-Q., 2015, Application of improved calculation method of Rayleigh damping coefficients to seismic response analysis on quay crane structure, Journal of South China University of Technology (Natural Science), 43, 103-109 
12. PAN D.-G., 2013, An optimization solution for Rayleigh damping coefficients in seismic response analysis, Gongcheng Lixue/Engineering Mechanics, 30, 15-20

13. Pan D.G., Chen G.D., Gao L.L., 2017, A constrained optimal Rayleigh damping coefficients for structures with closely spaced natural frequencies in seismic analysis, Advances in Structural Engineering, 20, 81-95

14. Park D., Hashash Y.M.A., 2004, Soil damping formulation in nonlinear time domain site response analysis, Journal of Earthquake Engineering, 249-274

15. Rayleigh J.W.S., Lindsay R.B., 1945, The Theory of Sound, 1st ed., New York: Dover Publications

16. Reda Taha M.M., Noureldin A., Lucero J.L., BacA T.J., 2006, Wavelet transform for structural health monitoring: a compendium of uses and features, Structural Health Monitoring, 5, 267-296

17. Tamura Y., Suganuma S.-Y., 1996, Evaluation of amplitude-dependent damping and natural frequency of buildings during strong winds, Journal of Wind Engineering and Industrial Aerodynamics, 59, 115-130

18. WAlha L., FAKhfakh T., HAdDAR M., 2009, Nonlinear dynamics of a two-stage gear system with mesh stiffness fluctuation, bearing flexibility and backlash, Mechanism and Machine Theory, 44, 1058-1069

19. Yassine D., Hammani A., Walha L., Haddar M., 2014, Effects of gear mesh fluctuation and defaults on the dynamic behaviour of two-stage straight bevel system, Mechanism and Machine Theory, 82, 71-86

20. Yousfi N., Zghal B., Akrout A., Walha L., Haddar M., 2018, Damping models identification of a spur gear pair, Mechanism and Machine Theory, 122, 371-388

21. Zhang H.D., WANG Y.F., 2010, Study on seismic time-history response of structures with complex damping, Gongcheng Lixue/Engineering Mechanics, 109-115 